\title{
巨猿究竟屬于猿的系統还是人的系統?
}

\author{
評吴汝康著“巨猿下領骨和牙齒化石”
}

安 志 敏

巨猿化石为解放后古生物学上的重要发 現之一。过去, 資产阶級的人类学家仅根据 在中䒺鋪里眴得的几枚牙齿就䚿为它們属于 一种新类型的猿，并且定名为“巨猿”，后又 有人改称为“巨人”。它們究竟属于猿的系統 还是人的系統，意見比較分岐，长期以来末 能获得解决。至于这些巨猿牙齿化石的出土 地、共生动物化石以及地稹年代，也都是不 很清楚的。从1956 年起, 中国科学院古脊椎 动物与古人类研究所在广西的来宾、上林、 武鳴、柳州、崇左、大新、扶䋁等县市, 調 查了 200 多个山洞，发現了三具巨猿下領骨 和許多牙齿化石，亚收集了 1,000 多枚牙齿 化石。在这样丰富賛料的基础上，不仅明确 了巨猿化石的性稹和出土地点，同时根据它 們的共生动物化石也解决了地稹年代的問 題，尽管目前的意見占不統一，一般都䚿为 应属于早更新世或上新世晚期, 最晚也应属 于中更新世初期。根据共生的动物化石进行 系統研究以后, 这个問題是不难确切解决 的。

自从开始調查发掘以来, 发表了不少有 关的报告或論文。吳汝康著 “巨猿下領骨和 牙齿化石”(中国古生物志，总号第 146 册，
新丁种 11 号,科学出版社 1962 年 9 月出版) 一书, 在过去研究的基础上, 对巨猿化石的 全部資料，作了細致的描述、精密的測量和 系統的分析研究。全书除引言外, 共由三个 部分所組成，今簡单介紹其內容如下：

“引言”对巨猿化石的发現历史、研究情 况及其存在問题等作了概括的叙述。

第一部分为“巨猿的下領骨”，包括对广 西楖城第一号洞所发現的三个下領骨材料的 叙迅、年龄和性別的鉴定、形态的覌察、測 量的指数、下領枝的复原和下領骨的特征等 节。作者充分利用了巨猿的杍料同現代猿、 猿人和現代人相比較，說明了它們的某些特 征接近猿类, 而另外一些特征又接近于化石 人类，基本上是介乎猿类与人类之間。

第二部分“巨猿的牙齿”对于发掘和收集 的 1,094 枚牙齿作了分析和研究, 也同样包 括了材料的叙述、形态的描述、牙齿萌出的 順序、牙齿的大小比例以及牙齿的特征等五 节。作者从比較材料上說明，巨猿牙达的形 态也介于猿类与人类之間，但某些特征更偏 近于人类。

第三部分为 “巨猿在分类系統中的地位 及其生活习性等問題”，其中包括了巨猿在 
灵长类系統中的地位，巨猿分类中存在的一 些問題，巨猿的体态，巨猿的生活习性，巨 猿的个体数目、两性数目和年龄統計, 巨猿 的牙病，巨猿的研究与“劳动創造人类”理論 关系等七节。全面叙述了作者对于巨猿化石 及其有关問題的理解，特別闡述了关于人类 发展理論的一些見解。作者強調将人科分为 真人亚科和前人亚科两个亚科的意見。真人 能够制造工具, 有社会組織, 可称为社会 人; 前人已营地面生活, 近乎直立或完全直 立, 脑子还小, 虽能利用天然的木棒和石块 获取食物或作防御攻击之用，但还不能制造 工具，也沒有社会組織，可称为生物人。巨 猿郎前人阶段末期的一个旁枝。此外，还記 述了巨猿个体的数目以及性別、年龄和牙病 的比例, 对于巨猿的体态和生活习性等均作 了一定的推測。

这批資料在古生物学和人类学的研究上 都极为重要。本书通过詳密的覌察測量和系 統的研究, 作出了若干关鍵性的結論, 花費 了相当多的劳动, 也取得了一定的成果。不 过本书的第三部分, 在相当大的范围內牵涉 到目前学术界正在討論的最古人类的問題, 其中的若干論点, 我們靽不能完全同意, 准 备就本书所討論的范围內, 提出几点不成熟 的看法，作为継續討論的参考。

\section{一 巨猿在灵长类系統中的地位問題}

巨猿下領骨及牙迕化石的性稹，介于猿 类和人类之間，这个特征是比較肯定的。不 过作者似乎过多地強調了巨猿化石接近人类 的某些特征，而沒有充分重視它所具有的猿 类成分。其实, 无論从巨猿的牙齿以及下領 骨的特征上，都很难証实作者的論点。如从 牙冠的比例上, 下曰齿的后部跟㛗窄于前部
三角座。猿类祖先三角座的下前尖（大多数 的灵长类此尖已經消失)、下原尖与下后尖 排成三角形，在此点上立猿显然接近于猿类 而距人类較远，因为人牙的后部寬于前部， 与猿类不同。巨猿田齿的下內尖与下次小尖 的后面边緣上有所謂第六尖，它属于森林古 猿的典型特征, 而且在主要的几个尖的安排 上也与森林古猿接近，可以証明巨猿与猿类 接近。另外，巨猿的下第三曰齿沒有显出退 化的現象，也与人类不同 ${ }^{[1]}$ 。但作者过份強 調了巨猿牙齿具有人的性稹：“猿类的下第 一自齿沒有第六齿尖，而在人类則常有之， 巨猿下領骨 II 的左右 $M_{1}$ 都有第六齿尖，特 別是在左側为明显。下領骨 I 和 III 由于田 齿已有較大的磨耗，不能辨別，这点也表示 巨猿接近人类”(原书 49 頁，下同）。在过去 的交献中，华南的化石猖猖 (Orang) 的左下 第一曰齿曾有第六尖，近代人类中則极为罕 見，仅有个別的例子，同时在中国猿人牙齿 里，第六尖也被視为接近猿类的原始特征之 一ㄹㅇㅇ 据作者說: “巨猿下第一、二曰齿的 标本虽有 150 个以上 ……在下中尖与下內尖 之間有第六尖存在, 占全部标本的 $26 \%$ "(36 頁)。在提到下第三白齿时說: “ 31 个标本 上,有第六副尖存在,介于下內尖和下中尖之 間，这种情况占全部标本的 $28 \%$ "(37 頁)。 根据以上的数字，說明巨猿白齿中第六尖的 存在比較普遍, 在这一点上, 它和人类也是 比較疏远的。至于下領骨的形态, 也同样接 近于猿类。如下領联合的矢状切面的輪廊与 現代猿类十分相象; 下領齿弓呈U字形，齿 弓的指数較大，甚至于淩駕于一切猿类之 上 ${ }^{[3]}$ 。根据以上各点, 我們䚯为巨猿接近人 类的說法不能成立,也不能同意把它列为“前 人亚科”的主张。把它列入猿科而另辟一“巨 
猿亚科”的意見 ${ }^{[4]}$ ，我們䚿为是比較妥当的。

\section{二 巨猿的体态問題}

1. 巨猿能否直立行走?

作者基于： “由于直立姿势而引起身体 結构上一系列的变化，犬齿和門齿变小了， 牙齿的大小在齿列上由前向后減小的現象， 在人类进化过程中是很明显的，随着前部牙 齿的減小，面部突領的程度也減小了”（56 頁)的假設，进而肯定: “可以推測巨猿可能 已經能够作一定程度的两足行走” (55 頁)。 我們覚得这个論断的証据是不充分的, 因为 直立与否, 决不能单純根据牙齿的形态大小 和面部突領的減小程度，而应該結合头骨、 骨徰和四肢骨的結构性稹来制断。如作者列 入前人阶段的南方古猿，人类学家一致同意 它已能直立行走，主要是根据它的头骨、骨 缊和下肢骨的各項特征而予以肯定的，但巨 猿缺乏以上的材料，論据显然不足。同时从 巨猿化石的性貭上分析，已有人指出：“巨 猿下領骨前部傾角很尖銳 $\left(52-56^{\circ}\right)$ 。两性 差异十分明显，雄性成年个体有显明的齿間 隙(第三下頜)。……在牙斗方面也同样具有 一系列猿类特点” ${ }^{[5]}$ 。作者也曾提出: “魁人 的下犬齿則比南方古猿中最大的犬齿还大。 而巨猿下犬类則又大于魁人” (26 頁)。除此 以外，“巨猿的第一下前曰齿，在其近中煩 面釉稹明显的向下延伸，形成一斜面，用以 接受上犬齿的胶合”[16] 根据以上的諭述，則 巨猿上頜的牙列形状当与一般猿类没有多大 的差异，因此，我們非常怀疑，巨猿头骨的 形态是否会接近于南方古猿而不同于一般猿 类？更不能設想巨猿已达到南方古猿那样直 立的程度。

只根据牙齿的縮小和突領的減小来推断
能否直立，这与过去所发現的灵长类化石的 情况也不相符合。在印度所发現的化石猿类 如拉瑪猿属(Ramapithecus)、布拉瑪猿属(Bramapithecus)和梭及立凡猿属(Sugrivapithecus)， 它們一般都是牙齿小、日齿构造簡单，齿列 也接近于人类，特別是拉桭古猿的上牙弓呈 抛物綫的形状，缺乏齿間隙，而且突領減 小，但是它們是否可以直立，迄今尚无确切 的証据可凭。

\section{2. 巨猿的身高}

作者推断巨猿: “身高可能与現代人相 似或稍大于現代人”(55 頁)，“由于巨猿属于 人的系統，可以推想巨猿有䡴长的股骨（55 頁)。只根据三个下領骨和 1,000 多枚牙齿来 估計巨猿的身高，未免超出現有材料之外。 我們同意作者的意見：“巨猿的确实身高只 有在发現其肢骨后才能解决”(55 頁)。不过 所提出的：“上領骨的大小必然与下領骨有 一定的关系。面部的全面高度(由鼻根点到頦 点的距离)可根据下領联合部的高度来推算。 巨猿下領骨 I 在联合处的高度为 64 毫米, 下領骨 III 为 85 毫米，根据人类的比例，則 其全面高大約各为 240 毫米和 320 毫米”(54 頁）的論据，是否符合于巨猿的实际全面高 度，是值得怀疑的。势必产生下列的問題： （1）巨猿下領的联合处的高度是否与全面的 高度有关? 它們的性稹和程度如何?（2）巨 猿下領骨的联合处的高度与人类的比例关系 如何？它們之間有无差异?（3）人类的下領 联合高与本身的全面高的关系如何? 因为巨 猿的下領形状并不和人类一致，似乎不能以 人类的比例来求巨猿的全面高。据 “下領骨 前部傾角的比較表” (10 頁，表 4)，巨猿下領 骨 $51.5-59^{\circ}$ ，而化石人类为 $60-64^{\circ}$ ，現 代人为 $75-91^{\circ}$, 可以推測它們的面部形状 
并不一致。郡末，用人类下領联合处高与全 面高的比例去推算巨猿的全面高，恐怕过于 簡单化了。同时据作者推算的結果，巨猿下 領 I 为 240 毫米,下領 III为 320 毫米, 比类人 猿面的全面高的 120.6-176.2 毫米的下限大 一倍到两倍，上限几乎大二分之一到一倍; 比中国猿人的全面高 118 毫米几乎大一倍到 两倍; 比近代人的全面高本均数值 125 毫米 几乎大一倍到两倍, 上述推算似乎是不合理 的。表 2 “巨猿下領骨各部的高度和厚度”(4 頁）和表 10 “巨猿下領骨測量总表” (19 頁) 的联合处高度数字不同，前者下領骨 I 65、 下領骨 II 62、下領 III 91，而后者下領骨 I 64、下領骨 II 60.5 、下領骨 III 85 ，疑系排 印的錯誤。

关于巨猿脑量的数值，作者推測: “可 能在 700一800 毫升左右”(54 頁), 根据也同 样是不充分的。

\section{三 巨猿的生活习性問題}

关于巨猿的生活习性；除从化石的特点 上覌察外，也还应該根据堆积的情况来考 虑，不幸的是从已发表的材料中对这一点还 不够十分清楚。作者訓为: “巨猿洞有几个 很小的縮寉区，不能容納躯体碩大的巨猿在 洞里活动，同时巨猿不是居住在洞內的。牙 齿化石在洞的水平分布上，两側比中部为 多，这表明水流在巨猿洞的堆积的形成中可 能起了一定的作用”(56 頁)。但据发現人的 推断: “巨猿和其他动物可能轓在这个洞內 居住过，它們死后，遺䯚就埋藏在这些堆积 之中" [7]，有的分析則訩为: “在巨猿洞保存 的犀类和象类多为乳类，說明它們死亡于幼 年阶段的較多，而这些身体庞大的动物，它 們是不居住在洞穴內的, 要訩为它們都死亡
在洞內, 势必不可能, 因而, 它們可能是 被其他动物如虎、豹等大型食肉类所捕杀， 尤其是它們的幼年者較之青年或成年者要軟 弱一些, 更容易成为食肉类的捕获物, 而猪 类更是随时可能成为食肉动物的牺牲品。所 以，以上三类动物，很可能是被捕后拖进洞 內的……食肉类所吃滕下的东西, 也可能被、 謷狗带入洞內” [8]。根据后两种的分析，則 巨猿洞的准积不象是由于流水作用形成的。 我們同意作者的分析, 巨猿是杂食者, 洞里 的动物化石也不可能是被巨猿带入洞內作为 食物的。那么，根据共生动物化石的性稹 来分析，势必承訓巨猿也是肉食动物的捕获 品, 由別处带到洞內。另外, 从只发現下領 骨和牙些而不見肢骨化石，更証实巨猿被作 为粞性品的可能性是相当大的。如果巨猿不 住在山洞里, 它們的活动区域也是值得探索 的。根据猿类的习性, 以活动于森林地带的 可能性为最大, 如果巨猿真的是习慣于森林 生活, 那么能否象人一样地直立行走, 恐怕 也需要重新考虑了。

\section{四 关于“生物人”和“社会人”的問題}

作者同意某些人类学家的意見: “我們 同意把人科分为两个亚科; 即真人亚科和前 人亚科。真人亚科包括能制造工具的人, 开 始有社会組織, 所以我們䚯为可以叫社会 人(Social man); 前人亚科包括营地面生活、 能近乎直立或完全值立，但脑子还較小，虽 能利用自然的木棒和石块等来获取食物或作 防御或攻击之用，但还不能用石块等制造工 具，因而也还没有社会組織，我們䚿为可以 叫生物人 (Biological man)"(52 頁)。作者的 論点在已发表的論交 ${ }^{[9]}$ 和本书中都做了詳尽 的開述，但我們不能同意这个論点，更不同 
意用巨猿化石作为这个論点的有力支持。

关于人的定义，本来是沒有异議的，但 提出“生物人”和“社会人”以后, 势必增加一 定程度的混乱。馬克思主义經典作家向来把 人类形成和社会发生看作同一过程的两个方 面, 而訜为两者是不容分割的。如馬克思所 指出的: “但是人的本稹, 并不是单个人所 固有的抽象物, 实际上, 它是一切社会关系 的总和”[10]。由此可以証明人的形成过程， 首先是社会关系总合体的形成过程, 郎社会 的形成过程, 因此人类的形成和社会的发生 是同时的，决不可以划分为“生物人”与 “社 会人”。

同时在“劳动創造人类”的理論上，馬克 思主义經典作家已經指出了劳动在人类起源 中的决定性作用，并給人类和动物之間划分 了一条分界綫, 这个标准一直为科学界所共 同恪守。如恩格斯說: “手的专門化, 意味着 工具的出現, 而工具意味着人所特有的活动, 意味着人对自然界的有改造意义的作用; 意 味着生产”[11]。又說: “沒有一只猿手篔經制 造过一把郎使最粗笨的石刀”[12]。所指出的 界綫非常清楚。因此，“劳动創造人类”的学 說, 并不能作为“前人”或“生物人”的理論根 据。我們不同意原书对于經典著作所作的若 干解释 (59-60 頁); 特別是作者所提出的: “因而我們不难理解，恩格斯在写作这篇文 章时, 在科学材料的引用上, 受到了当时科 学水平的一定限制”(60 頁) 的說法, 不能訩 为是正确的。决不可能由于某些新的化石的 发現，就改变了“人”的定义。

\section{五 关于“天然工具”的問題}

作者过去所发表的一系列論交中，都坚 持了在人类能够制造工具以前，还有一个使
用“天然工具”的阶段，本书也多方面强調了 这个論点。如 “虽能利用自然木棒和石块等 来获取食物或作防御攻击之用，但还不能用 石块制造工具” (52 頁)，“因而游离的双手能 够比較便利地利用自然的木棍和石块, 可以 代替犬齿在防御和攻击中的作用” (55 頁)， “从而能更有效地使用木棍和石块等 来获取 食物和保卫自己，成了两条艆行走的前人” (60 頁)，“也需要充分发展双手使用各种“天 然工具” 的灵活性 ”(60 頁) 等。关鍵在于这 些未經加工的木棒、石块, 能否称为“工具”; 同时猿类在有些場合也会使用木棒或石块， 恩格斯早已指出这种現象: “許多猿类用手 在树上筑巢或者象黑猖猖一样在树枝間修造 住处以避风雨。它們用手拿着木棒抵御敌人 或者以果实和石块向敌人投擲” [13]。許多人 类学家也都从实驗或覌察中証 实了这个事 实。从来沒有人把猿类所使用的木棒和石块 称为 “工具”，因为猿类既不能对它們加工制 造, 也不能經常使用它們。如果按着“前人” 的定义，現代的类人猿至少也具有某些 “前 人”的因素了, 因此, 所謂“生物人”的說法 是不可能成立的。我們应該根据恩格斯的科 学定义, 以能够制造工具为人和猿的分界标 准。不拘南方古猿也好, 巨猿也好, 只要它 們能制造工具, 就属于人的范畴; 否則它們 仍属于猿的系統，而不能称其为 “前人” 或 “生物人”。关于 “劳动創造人类” 理諭的研 究, 属于社会科学的范畴, 为阶級斗爭的有 力武器, 决不应該只用生物学的覌点来解释 它。

关于巨猿能否使用“天然工具”，也还沒 有直接的証据。如作者所說明的: “虽然至 今还沒有发現巨猿的肢骨，但从整个前人阶 段各种类型的体貭結构上，可以推測巨猿可 
能已經能够作一定程度的两足行走，因而游 离的双手能够比較便利地利用自然的木棒或 石块，可以代替犬崡在防御和攻击中的作 用”(55頁)。“为了要使木棍和石块在他們手 中能发揮充分的效用, 需要有較大的身材, 也还需要充分发揮双手使用各种 '天然工具” 的灵活性” (60 頁)。我們覚得这些假設都难 于成立，并不能因为巨猿的犬齿不发达或有 較大的身材，就証明它們已經能够使用 “天 然工具”。

至于巨猿的絕灭原因，作者曾解释为: “巨猿的身体向着极度巨大的方向发展时,需 要的营养增多，身体的結构随着愈为特化， 这种情形下，其繁殖能力也会減低，而且在 改变食性上会有更大的困难, 因而一旦外界 的生存条件 (如气候、植物等) 有較大改变 时, 便易于絕灭” (56 頁) 的說法, 也不見得 全面。前面已經分析过, 从山洞內所发現的 巨猿下領骨及牙齿化石，显然是食肉类动物 的捕获品，甚至于如巨猿一洞所发現的至少 代表 77 个个体（原书 57 頁作 72 个个体，与 表 37 的数字不符，雌雄个体的数目也有出 入，疑其中的一处是錯誤的），可能說明它 們是比較缺乏防御力量的。由于巨猿缺之和
自然作斗爭的能力, 正是它走向絕灭的主导 原因之一。我們很难想象巨猿們能够經常地 利用天然的木棒和石块进行防御和攻击, 并 利用它們来取得生活資料; 更不能想象在巨 猿的生活中，已具有人类的任何因素。

[1] Nesturkh M. F., The Orign of Man, p. 89, 1959.

[2] Weidenreich F., The dentition of Sinanthropus Pekinensis, Palaeontologia Sinica, New Series D, No. 1, 1937, p. 85, 86,89, 153.

[3] 董悌忱, 古脊椎动物与古人类, 6 卷 4 期, 376 頁, 图 1、2,1962。

[4]同上, 381頁。

[5]同上, 376 頁。

[6]同上, 378 頁。

[7]裴文中、本有㥀, 古脊椎动物学报, 2 卷 4 期, 194 面, 1958 年。

[8] 韓德芬, 古猆椎动物与古人类, 2 卷 1 期, 76 面, 1960 年。

[9] “从“曙石器”問題談到中国猿人是否是最早的人”, 光明日报，1961 年9月6日; “我国古人类学的新 进展”，科学通报，1962 年 8 期; “关于人类和猿类 化石最近的砰究成果”，人民日报，1962 年 8 月 25 日。

[10] 馬克思恩格斯全集, 第三卷, 5 頁, 人民出版驵, 1960 年。

[11] 恩格斯, 自然䞑証法，15 面，人民出版社，1955 年。

[12]同上, 138 頁。

[13] 同上, 138 頁。 